\title{
GaSb/AIAsSb Resonant Tunneling Diodes with GaAsSb Emitter Prewells
}

\author{
Andreas Pfenning, ${ }^{1, a)}$ Georg Knebl, ${ }^{1}$ Fabian Hartmann,,${ }^{1, b)}$ Robert Weih, ${ }^{1}$ Manuel Meyer, ${ }^{1}$ Andreas \\ Bader, ${ }^{1}$ Monika Emmerling ${ }^{1}$, Lukas Worschech ${ }^{1}$, Sven Höfling, ${ }^{1,2}$ \\ 1) Technische Physik, Physikalisches Institut and Röntgen Center for Complex Material Systems (RCCM), Universität \\ Würzburg, Am Hubland, D-97074 Würzburg, Germany \\ 2) SUPA, School of Physics and Astronomy, University of St. Andrews, St. Andrews, KY16 9SS, United Kingdom
}

We investigate the electronic transport properties of GaSb/AIAsSb double barrier resonant tunneling diodes with pseudomorphically grown ternary $\mathrm{GaAs}_{x} \mathrm{Sb}_{1-\mathrm{x}}$ emitter prewells over a broad temperature range. At room temperature, resonant tunneling is observed and the peak-to-valley current ratio (PVCR) is enhanced with increasing As mole fraction from $1.88\left(\mathrm{GaAs}_{0.07} \mathrm{Sb}_{0.93}\right.$ prewell), to 2.08 ( $\mathrm{AAs}_{0.09} \mathrm{Sb}_{0.91}$ prewell) up to 2.36 (GaAs $0.11 \mathrm{Sb}_{0.89}$ prewell). The rise in PVCR is attributed to an enhanced carrier density at the $\Gamma$-valley within the emitter prewell. On the contrary at cryogenic temperatures, increasing the As mole fractions reduces the PVCR. At a temperature of $T=4.2 \mathrm{~K}$, reference samples without incorporation of an emitter prewell containing As show PVCRs up to 20.4. We attribute the reduced PVCR to a degraded crystal quality of the resonant tunneling structure caused by As incorporation and subsequently an enhanced defect scattering at the interfaces.

a) Electronic mail to: Andreas.Pfenning@physik.uni-wuerzburg.de

b) Electronic mail to: Fabian.Hartmann@physik.uni-wuerzburg.de

The three semiconductors AISb, GaSb and InAs form a nearly lattice-matched set at a lattice constant of about $6.1 \AA$ and cover a wide range of bandgap energies and band line-ups. ${ }^{1} 6.1 \AA$ semiconductors have been exploited to realize a variety of electronic and optoelectronic devices, such as high-mobility transistors, resonant tunneling diodes (RTDs), as well as mid-infrared (MIR) light sources and detectors. ${ }^{2-4}$ Recently, we proposed to transfer the resonant tunneling diode photodetector principle to the MIR spectral region, by combining a GaSb/AISb double barrier resonant tunneling structure with a narrow bandgap absorption region. ${ }^{5}$ The RTD photodetection principle provides high internal carrier amplification at considerably low operation voltages, ${ }^{6-9}$ and is based on a large resonant tunneling current, that is modulated by photogenerated minority charge carriers. ${ }^{10,11}$ Besides the amplification of optically generated charge carriers, alternative sensor schemes and operation modes can be utilized in RTD photodetectors that exploit the region of negative differential conductance (NDC). The NDC region provides the means to use stochastic resonance principles and to operate RTDs as optoelectronic switches. ${ }^{12-14}$ Unfortunately most RTDs and resonant interband tunneling diodes (RITDs) of the $6.1 \AA$ family are poorly suited as photodetectors because of their staggered or even broken bandgap alignment. Although these tunneling diodes show remarkable electronic properties with peak-to-valley current ratios above 20 at room temperature and an aptitude for RTD high frequency applications, the bandgap 
alignment of InAs and $\mathrm{Al}(\mathrm{As}) \mathrm{Sb}$ barrier structures does not provide the means for sufficient accumulation of minority and photogenerated charge carriers as required for opto-electronic applications of antimonide-based RTDs. ${ }^{2}$ However, the AISb/GaSb double barrier resonant tunneling diode has a type-I bandgap alignment with large conduction and valence band offsets, which provides the means of minority charge carrier accumulation. ${ }^{15}$

In a recent study, we demonstrated room temperature resonant tunneling of electrons in AlAsSb/GaSb double barrier RTDs by exploiting pseudomorphically grown emitter prewell structures comprising the ternary compound semiconductors GalnSb and GaAsSb. Peak-to-valley current ratios (PVCR) of 1.45 (GalnSb prewell) and 1.36 (GaAsSb prewell) were observed. The main purpose of the emitter prewells is to increase the $\Gamma$ - $L$ valley energy separation and to provide a repopulation of $\Gamma$-electrons in the emitter. To further improve the PVCR we proposed to increase the In or As mole fractions of the prewell. ${ }^{16}$

Here, we present a study on the electronic transport properties of AIAsSb/GaSb double barrier RTDs with pseudomorphically grown $\mathrm{GaAs}_{\mathrm{x}} \mathrm{Sb}_{1-\mathrm{x}}$ emitter prewell structures with increasing As mole fractions from $x=7 \%$ up to $x=11 \%$. At room temperature, the resonance current density is found to increase with larger As mole fractions, which leads to enhanced peak-to-valley current ratios of PVCR $=1.88(x=7 \%), 2.03(x=9 \%)$, and $2.36(x=11 \%)$. At cryogenic temperatures, increasing As mole fractions however reduce the PVCR. At $T=$ $4.2 \mathrm{~K}$, reference samples without incorporation of an emitter prewell containing As show PVCRs up to 20.4, whereas for an RTD with a prewell As mole fraction of $x=11 \%$, the PVCR is as low as 3.9. We attribute the reduced PVCR to a degraded crystal quality of the resonant tunneling structure caused by As incorporation and subsequently an enhanced defect scattering at the interfaces.

The samples are grown by means of molecular beam epitaxy (MBE) on an n-type Te-doped GaSb (100) substrate. Sample growth starts with $300 \mathrm{~nm}$ of $\mathrm{n}$-type Te-doped GaSb with a doping concentration of $n=$ $1 \times 10^{18} \mathrm{~cm}^{-3}$, followed by $120 \mathrm{~nm}$ of GaSb with a reduced doping concentration of $n=5 \times 10^{17} \mathrm{~cm}^{-3}$. Subsequently, the undoped resonant tunneling structure is grown, starting with $20 \mathrm{~nm}$ of GaSb and a pseudomorphically grown, ternary $\mathrm{GaAs}_{\mathrm{x}} \mathrm{Sb}_{1-\mathrm{x}}$ emitter prewell. The $5 \mathrm{~nm}$ wide main quantum well is also comprised of the ternary $\mathrm{GaAs}_{x} \mathrm{Sb}_{1-\mathrm{x}}$ and sandwiched between two lattice-matched and $4.5 \mathrm{~nm}$ thick $\mathrm{AlAs}_{0.08} \mathrm{Sb}_{0.92}$ barriers. The As mole fractions of the emitter prewell and the main quantum well are $x=7 \%$ (RTD-1), $x=9 \%$ (RTD-2) and $x=11 \%$ (RTD-3). The resonant tunneling structure is finalized by $27 \mathrm{~nm}$ of GaSb. To form the collector-side contact region, $120 \mathrm{~nm}$ of GaSb with $n=5 \times 10^{17} \mathrm{~cm}^{-3}$ are grown, followed by lattice-matched $220 \mathrm{~nm} \mathrm{Al} \mathrm{l}_{0.3} \mathrm{Ga}_{0.7} \mathrm{As}_{0.03} \mathrm{Sb}_{0.97}$ optical window and contact region with $n=1 \times 10^{18} \mathrm{~cm}^{-3}$. A $10 \mathrm{~nm}$ wide GaSb capping layer with $n=1 \times 10^{18} \mathrm{~cm}^{-3}$ completes the growth process. Further and more detailed information on the growth and fabrication process can be found in reference [16].

Figure 1 (a) shows the simulated $\Gamma$-point conduction band (CB) profile of the resonant tunneling structures (RTS) of the investigated samples RTD-1 (black), RTD-2 (red) and RTD-3 (green). The simulation is performed with the nextnano software for simulation of electronic and optoelectronic semiconductor devices. ${ }^{17}$ The incorporation of As reduces the energy of the $\mathrm{CB}$ at the $\Gamma$-point, which leads to an increasing prewell depth of $\Delta E_{\Gamma}=80 \mathrm{meV}, 102 \mathrm{meV}$ and $124 \mathrm{meV}$ for RTD-1, RTD-2 and RTD-3, respectively. Figure 1 (b) and (c) show the electron density profiles for electrons occupying the $L$ - and $\Gamma$ - valley, respectively. For increasing As mole 
fractions, the electron density at the L-point remains nearly constant, whereas the electron density at the $\Gamma$ point increases. This confirms, yet, also clarifies our previous claim, in which we attributed the improved PVCRs to a depopulation of L-valley states with a simultaneous repopulation of the $\Gamma$-valley. Apparently for RTDs with GaAsSb emitter prewells, the population of L-valley states persist and the electron density stays almost constant. Yet, the $\Gamma$-valley is efficiently repopulated due to the increasing prewell emitter depth. As a result, the emitter prewell can provide sufficiently large quantities of $\Gamma$-electrons for resonant tunneling.

Cross-sectional scanning electron microscopy (SEM) and time-of flight secondary ion mass spectroscopy (TOF-SIMS) confirm the widths of the semiconductor layer structures and the doping profiles, respectively. Highresolution X-ray diffraction (HR-XRD) measurements confirm the mole fractions of the ternary semiconductor layers. Experimental HR-XRD scans of RTD-1, RTD-2 and RTD-3 are shown in Figure 2 (a) as solid black, red and blue line, respectively. The corresponding simulations of the X-ray diffraction patterns are shown in Figure 2 (b). XRD counts are plotted on the logarithmic scale versus the diffraction angle $\Delta \omega$, with the GaSb substrate peak centered at the diffraction angle position of $\Delta \omega=0^{\circ}$. Secondary diffraction patterns arise at higher angles, due to the tensile-strained GaAsSb prewell and quantum well. For increasing As mole fractions, and hence increasing tensile strain, the secondary peak shifts to higher angles. The amplitude is not affected, as the prewell and quantum well widths of the different samples are identical.

Circular RTD mesa structures with diameters from $d=3 \mu \mathrm{m}$ up to $13 \mu \mathrm{m}$ are defined by optical lithography and dry chemical etching. The RTDs are contacted in a two-terminal set-up on the substrate backside ( $\mathrm{AuGe} / \mathrm{Ni} / \mathrm{Au}$ ) and a ring-shaped Ti/Au on top of the RTD mesa. The backside contact is connected to ground. A Hewlett-Packard 3251 Universal Source is used to apply a bias voltage to the top contact. A Keithley 2000 Digital Multimeter is used to measure the current by measuring the voltage drop across a resistance in series. For detailed information on the measurement approach see Ref. [8].

Figure 3 (a) shows the room temperature current density-voltage (j(V)-) characteristics of RTD-1, RTD-2 and RTD-3. For positive bias voltages, electrons are injected from the emitter prewell. For all three samples, a well-pronounced resonance current density is apparent, followed by a region of negative differential conductance (NDC) and valley current region. The resonance occurs at voltages of $V_{\text {res }}=1.25 \mathrm{~V}, 1.45 \mathrm{~V}$ and $1.56 \mathrm{~V}$, for RTD-1, RTD-2 and RTD-3, respectively. The shift towards larger bias voltages with increasing prewell depth or width is in accordance with previous studies on AIGaAs/GaAs double barrier RTDs with InGaAs emitter prewells ${ }^{18}$. This can be explained by the thickness difference between the emitter prewell and quantum well that leads to a larger shift of the prewell ground state energy compared to the quantum well ground state as the As mole fraction increases. The current density peaks when the emitter prewell and main quantum well states are aligned energetically ${ }^{18}$.

Besides $V_{\text {res }}$, also the resonance and valley current densities $\left(j_{\text {res }}\right.$ and $\left.j_{v a l}\right)$ increase from RTD-1 to RTD-3. Figure 3 (b) shows $j_{\text {res }}$ (black spheres) and $j_{v a l}$ (red spheres) as a function of As mole fraction. From the linear fit functions, we find that $j_{\text {res }}$ and $j_{v a l}$ increase by $(224 \pm 11) \mathrm{nA} \mu \mathrm{m}^{-2} \%^{-1}$ and $(65 \pm 16) \mathrm{nA} \mu \mathrm{m}^{-2} \%^{-1}$, respectively. This is in similar to previous studies performed on AlGaAs/GaAs double barrier RTDs with InGaAs emitter prewells. ${ }^{18-20}$ The linear fit functions of $j_{\text {res }}$ and $j_{v a l}$ cross at a mole fraction of $x \approx 3.7 \%$ suggesting this to be 
the minimum As mole fraction to observe resonant tunneling at room temperature. Due to $j_{\text {res }}>j_{v a l}$ with $(224 \pm 11) n A \mu m^{-2} \%^{-1}$ and $(65 \pm 16) n A \mu m^{-2} \%^{-1}$, the PVCR is enhanced with increasing As mole fractions. Figure 3 (c) shows the experimentally obtained PVCR (black spheres) versus mole fraction. The PVCR increases from PVCR $=1.88$ (RTD-1, $x=7 \%$ ) to 2.08 (RTD-2, $x=9 \%$ ) up to 2.36 (RTD-3, $x=11 \%$ ). The dashed line corresponds to extrapolation of the PVCR for higher and lower mole fractions and is obtained by dividing the linear fit functions of $j_{\text {res }}$ by $j_{v a l}$ (see Figure $3(\mathrm{~b})$ ). For a better comparison, the PVCRs for RTDs from Ref. [16] with $\mathrm{GaAs}_{0.05} \mathrm{Sb}_{0.95}$ (red square) and $\mathrm{Ga}_{0.84} \mathrm{In}_{0.16} \mathrm{Sb}$ prewell (blue diamond) are added to the graph. The PVCR of the RTD with $\mathrm{GaAs}_{0.05} \mathrm{Sb}_{0.95}$ emitter prewell is in good agreement with the extrapolation. We expect achievable room temperature PVCRs up to $P V C R \lesssim 3$.

To study the effect of an increasing As mole fraction on the RTS quality, two reference samples R-1 and R-2 are grown. In both samples, the ternary prewell and main quantum well are substituted by the binary GaSb. R-1 contains binary AISb barriers, whereas R-2 contains barriers of the lattice-matched $\mathrm{AlAs}_{0.08} \mathrm{Sb}_{0.92}$. Temperature series of $\mathrm{j}(\mathrm{V})$-characteristics are taken in a He flow cryostat, starting at $T=300 \mathrm{~K}$, reducing the temperature in steps of $\Delta T=25 \mathrm{~K}$ down to $T=4.2 \mathrm{~K}$. Figure 4 (a) shows the j(V)-characteristics of RTD-1 at room temperature (red, $T=300 \mathrm{~K}$ ) and $T=4.2 \mathrm{~K}$ (blue). At $T=4.2 \mathrm{~K}$ and compared to the room temperature $\mathrm{j}(\mathrm{V})$-characteristics, the resonance current density is enhanced, the valley current density reduced and the valley region is more pronounced. Yet, with a low-temperate value of only PVCR $=4.95$, the resulting lowtemperature peak-to-valley current ratio is reduced by a factor of 2 compared to values reported for structures without prewell. ${ }^{15,16}$

Figure 4 (b) shows the j(V)-characteristics of reference sample R-1 at room temperature (red, $T=300 \mathrm{~K}$ ) and at $T=4.2 \mathrm{~K}$ (blue). At room temperature, a little shoulder at $V=1.6 \mathrm{~V}$ indicates, the point of resonance. Yet, no region of negative differential conductance can be observed. At cryogenic temperatures of $T=4.2 \mathrm{~K}$, the $\mathrm{j}(\mathrm{V})$-characteristic is bistable and shows a well pronounced resonance current and valley current region. The resonance and valley current density are $j_{\text {res }}=960 \mathrm{nA} \mu \mathrm{m}^{-2}$ and $j_{v a l}=47 \mathrm{nA} \mu m^{-2}$, respectively. Hence, the peak-to-valley current ratio is $P V C R=20.4$. The PVCR is improved by a factor of four compared to RTD-1 and almost a factor of three compared to previously reported values. ${ }^{16,15}$

Figure 4 (c) shows the PVCR of R-1 (black spheres), R-2 (red circles), RTD-1 (green up-facing triangles) and RTD-3 (blue down-facing triangles) as a function of temperature from $T=4.2 \mathrm{~K}$ up to $T=300 \mathrm{~K}$. For all samples, the PVCR decreases with increasing temperature. At room temperature, the resonant tunneling structures containing the highest concentration of As show the largest PVCR, as caused by the successive population of the $\Gamma$-valley with electrons. At low temperatures, the highest PVCR of 20.4 is observed for a resonant tunneling structure without As. When As is incorporated in the resonant tunneling structure, the PVCR decreases. Without prewell but lattice-matched $\mathrm{AlAs}_{0.08} \mathrm{Sb}_{0.92}$ tunneling barriers, the PVCR decreases significantly down to PVCR $=$ 8.9 (R-2). With an As-containing ternary $\mathrm{GaAs}_{x} \mathrm{Sb}_{1-\mathrm{x}}$ emitter prewell, main quantum well and lattice-matched $\mathrm{AlAs}_{0.08} \mathrm{Sb}_{0.92}$ tunneling barriers, the PVCR is further reduced from PVCR $=4.9$ (RTD-1, $x=7 \%$ ) to PVCR $=3.9$ (RTD-3, $x=11 \%)$. 
For reference sample R-1, the extremely low valley current density together with the pronounced valley region is particularly striking, and indicates exceptionally good crystal quality of the RTS with few defects. At cryogenic temperatures, many of the transport channels that contribute to the valley current density, such as thermionic emission or tunneling through higher levels are canceled out. As a result, $j_{v a l}$ is mainly determined by sidewall leakage and inelastic scattering processes (e.g. impurity scattering, interface roughness scattering). ${ }^{21}$ Hence, we attribute the low valley current to a superior crystal quality and lower defect density throughout the resonant tunneling structure compared to As-containing resonant tunneling structures.

In conclusion, increasing the As mole fraction of pseudomorphically grown, ternary $\mathrm{GaAs}_{x} \mathrm{Sb}_{1-x}$ emitter prewells, leads to enhanced room temperature peak-to-valley current ratios of AlAsSb/GaSb RTDs. Room temperature PVCRs of up to 2.36 have been demonstrated for RTDs with $\mathrm{GaAs}_{0.11} \mathrm{Sb}_{0.89}$ prewell. This is attributed to a repopulation of $\Gamma$-valley electron states, which results from a reduced $\Gamma$-point conduction band energy of the emitter prewell. Besides of an enhanced room temperature PVCR, increasing the As mole fraction also degrades the structural quality of the RTS, which leads to decreasing PVCRs at cryogenic temperatures, caused by an enhanced defect scattering at the interfaces. The study shows a way how to enable RTD-based optoelectronic switches, ${ }^{12,14}$ or optically modulated oscillators. ${ }^{22,23}$ Fundamental characteristics and limitations of GaSb-based resonant tunneling structures are discussed, which might provide important insights for research groups working on more complex optoelectronic devices such as interband cascade detectors..$^{24,25}$

\section{Acknowledgements}

The authors are grateful for financial support by the state of Bavaria, and the German Ministry of Education and Research (BMBF) within the national project HIRT (FKZ 13XP5003B). 


\section{References}

${ }^{1}$ H. Kroemer, Phys. E Low-Dimensional Syst. Nanostructures 20, 196 (2004).

2 B.R. Bennett, R. Magno, J.B. Boos, W. Kruppa, and M.G. Ancona, Solid. State. Electron. 49, 1875 (2005).

${ }^{3}$ I. Vurgaftman, R. Weih, M. Kamp, J.R. Meyer, C.L. Canedy, C.S. Kim, M. Kim, W.W. Bewley, C.D. Merritt, J. Abell, and S. Höfling, J. Phys. D. Appl. Phys. 48, 123001 (2015).

${ }^{4}$ P. Martyniuk, M. Kopytko, and A. Rogalski, Opto-Electronics Rev. 22, 121 (2014).

${ }^{5}$ S. Höfling, A. Pfenning, R. Weih, A. Ratajczak, F. Hartmann, G. Knebl, M. Kamp, and L. Worschech, in edited by M. Strojnik (2016), p. 997306.

${ }^{6}$ W. Wang, Y. Hou, D. Xiong, N. Li, W. Lu, W. Wang, H. Chen, J. Zhou, E. Wu, and H. Zeng, Appl. Phys. Lett. 92, 23508 (2008).

${ }^{7}$ A. Pfenning, F. Hartmann, M. Rebello Sousa Dias, F. Langer, M. Kamp, L.K. Castelano, V. Lopez-Richard, G.E. Marques, S. Höfling, and L. Worschech, Appl. Phys. Lett. 107, 81104 (2015).

${ }^{8}$ A. Pfenning, F. Hartmann, F. Langer, M. Kamp, S. Höfling, and L. Worschech, Nanotechnology 27, 355202 (2016).

${ }^{9}$ A. Pfenning, F. Hartmann, F. Langer, S. Höfling, M. Kamp, and L. Worschech, Appl. Phys. Lett. 104, 101109 (2014).

${ }^{10}$ P. England, J.E. Golub, L.T. Florez, and J.P. Harbison, Appl. Phys. Lett. 58, 887 (1991).

${ }^{11}$ B. Romeira, L.M. Pessoa, H.M. Salgado, C.N. Ironside, and J.M.L. Figueiredo, Sensors (Basel). 13, 9464 (2013).

${ }^{12}$ T.S. Moise, Y.-C. Kao, L.D. Garrett, and J.C. Campbell, Appl. Phys. Lett. 66, 1104 (1995).

${ }^{13}$ O. Tretiakov and K. Matveev, Phys. Rev. B 71, 165326 (2005).

${ }^{14}$ F. Hartmann, L. Gammaitoni, S. Höfling, A. Forchel, and L. Worschech, Appl. Phys. Lett. 98, 242109 (2011).

15 J.L. Jimenez, X. Li, and W.I. Wang, Appl. Phys. Lett. 64, 2127 (1994).

${ }^{16}$ A. Pfenning, G. Knebl, F. Hartmann, R. Weih, A. Bader, M. Emmerling, M. Kamp, S. Höfling, and L. Worschech, Appl. Phys. Lett. 110, 33507 (2017).

${ }^{17}$ A. Trellakis, T. Zibold, T. Andlauer, S. Birner, R.K. Smith, R. Morschl, and P. Vogl, J. Comput. Electron. 5, 285 (2006).

${ }^{18}$ T.B. Boykin, R.C. Bowen, G. Klimeck, and K.L. Lear, Appl. Phys. Lett. 75, 1302 (1999).

${ }^{19}$ Y.W. Choi and C.R. Wie, J. Appl. Phys. 71, 1853 (1992).

${ }^{20}$ R.M. Lewis, H.P. Wei, S.Y. Lin, and J.F. Klem, Appl. Phys. Lett. 77, 2722 (2000).

${ }^{21}$ Jian Ping Sun, G. Haddad, P. Mazumder, and J.N. Schulman, Proc. IEEE 86, 641 (1998).

${ }^{22}$ T.J. Slight, B. Romeira, L. Wang, J.M.L. Figueiredo, E. Wasige, and C.N. Ironside, IEEE J. Quantum Electron. 44, 1158 (2008).

${ }^{23}$ J. Wang, A. Al-Khalidi, W. Zhang, E. Wasige, A.E. Kelly, S. Watson, H. Cantu, and J. Figueiredo, Third Int. Conf. Appl. Opt. Photonics 193 (2017).

${ }^{24}$ R.Q. Yang, Z. Tian, J.F. Klem, T.D. Mishima, M.B. Santos, and M.B. Johnson, Appl. Phys. Lett. 96, 63504 (2010).

${ }^{25}$ L. Lei, L. Li, W. Huang, J.A. Massengale, H. Ye, H. Lotfi, R.Q. Yang, T.D. Mishima, M.B. Santos, and M.B. Johnson, Appl. Phys. Lett. 111, 113504 (2017). 
Figure 1: (a) Simulation of the $\Gamma$-point conduction band (CB) profile of the resonant tunneling structure (RTS) of the samples RTD 1, RTD 2 and RTD 3, shown as black, red and green spheres, respectively. The emitter prewell and the main quantum well, which is sandwiched between two lattice-matched AlAsSb barriers, are comprised of the ternary compound semiconductor $\mathrm{GaAs}_{x} \mathrm{Sb}_{1-\mathrm{x}}$. The prewell and main quantum well depths increase with increasing As mole fraction. (b) Charge carrier density profiles of electrons occupying L-valley states of RTD-1, RTD-2 and RTD-3. (c) Charge carrier density profiles of electrons occupying $\Gamma$-valley states of RTD-1, RTD-2 and RTD-3.

Figure 2: High-resolution X-ray diffraction (HR-XRD) spectra of RTD-1 (black), RTD-2 (red) and RTD-3 (blue): (a) experimental data, (b) simulation. The HR-XRD spectra are centered at the GaSb substrate peak at $\Delta \omega=0^{\circ}$. Secondary diffraction patterns arise at positive diffraction angles due to the tensile strained emitter prewell and main quantum well. A larger shift corresponds to a higher As mole fraction.

Figure 3: (a) Room temperature current density-voltage characteristics for RTD-1 (green spheres), RTD-2 (red spheres) and RTD-3 (black spheres). (b) Resonance current density $\left(j_{\text {res }}\right.$, black spheres) and valley current density $\left(j_{v a l}\right.$, red spheres) as a function of As mole fraction. From the linear fit functions of $j_{\text {res }}$ and $j_{v a l}$, the minimum As mole fraction required to achieve room temperature resonant tunneling is determined to be $x \gtrsim 3.7 \%$. (c) Peak-to-valley current ratio (PVCR) as a function of As mole fraction. The results of this work are shown as black spheres. The dashed black line shows the extrapolation calculated from the linear fit functions from (b). Results from Ref. [16] are shown as red square ( $\mathrm{GaAs}_{0.05} \mathrm{Sb}_{0.95}$ emitter prewell) and blue diamond ( $\mathrm{Ga}_{0.86} \mathrm{In}_{0.16} \mathrm{Sb}$ emitter prewell).

Figure 4: (a) Current density-voltage characteristics of RTD-1 at room temperature ( $T=300 \mathrm{~K}$, red) and at $T=4.2 \mathrm{~K}$ (blue). (b) Current density-voltage characteristics of reference sample R-1 at room temperature ( $T=300 \mathrm{~K}$, red) and at $T=4.2 \mathrm{~K}$ (blue). (c) Peak-to-valley current ratio (PVCR) as a function of temperature for reference sample R-1 (black spheres), reference sample R-2 (red circles) as well as RTD-1 (green up-facing triangles) and RTD-3 (blue down-facing triangles). 

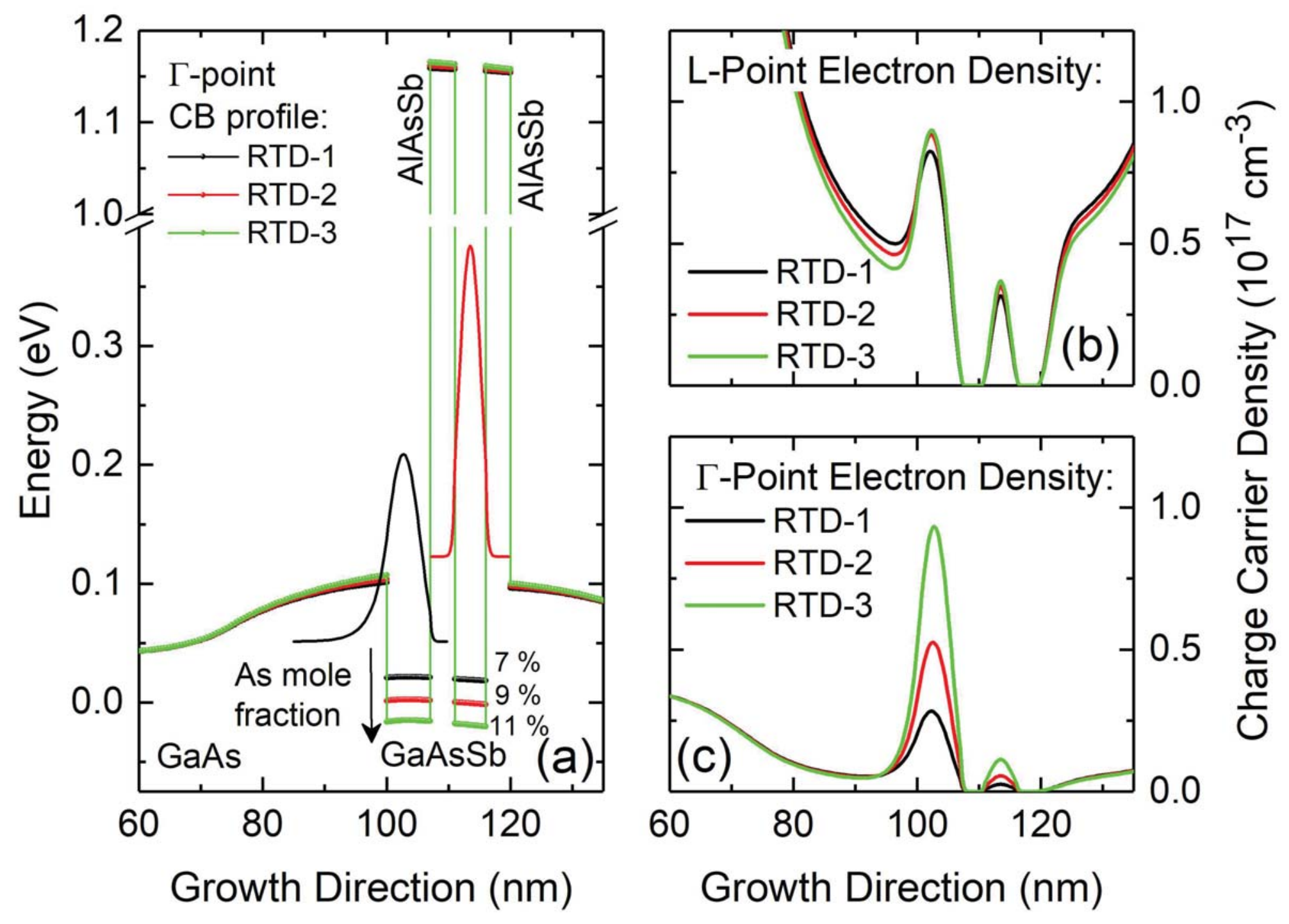


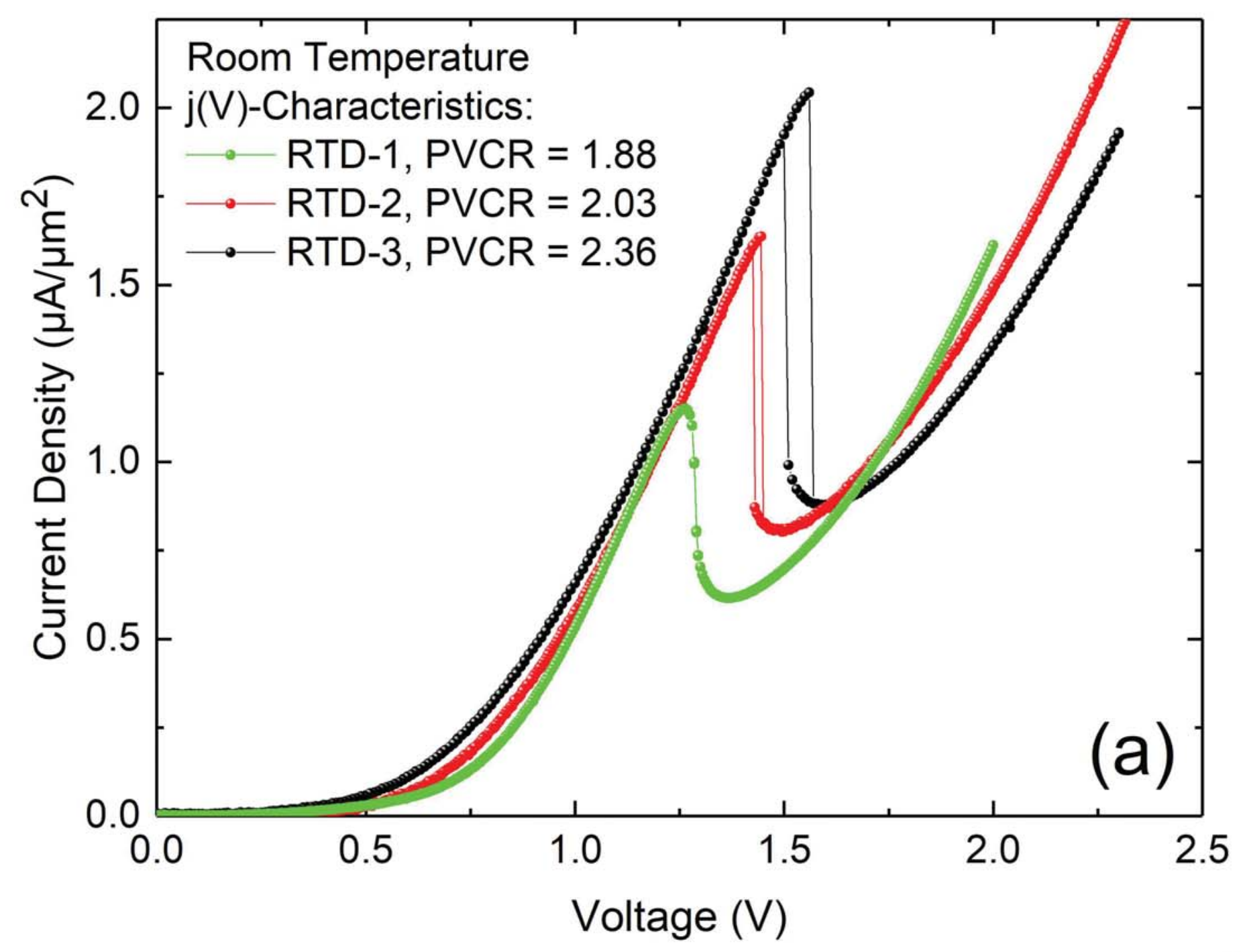

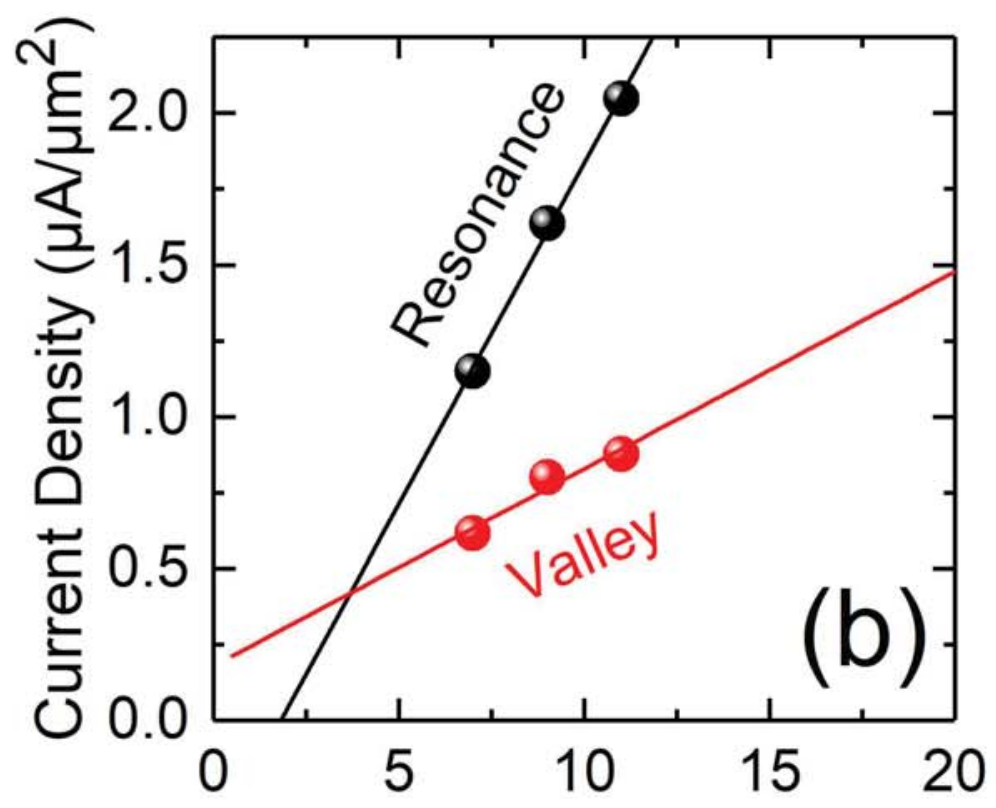

As Mole Fraction (\%)

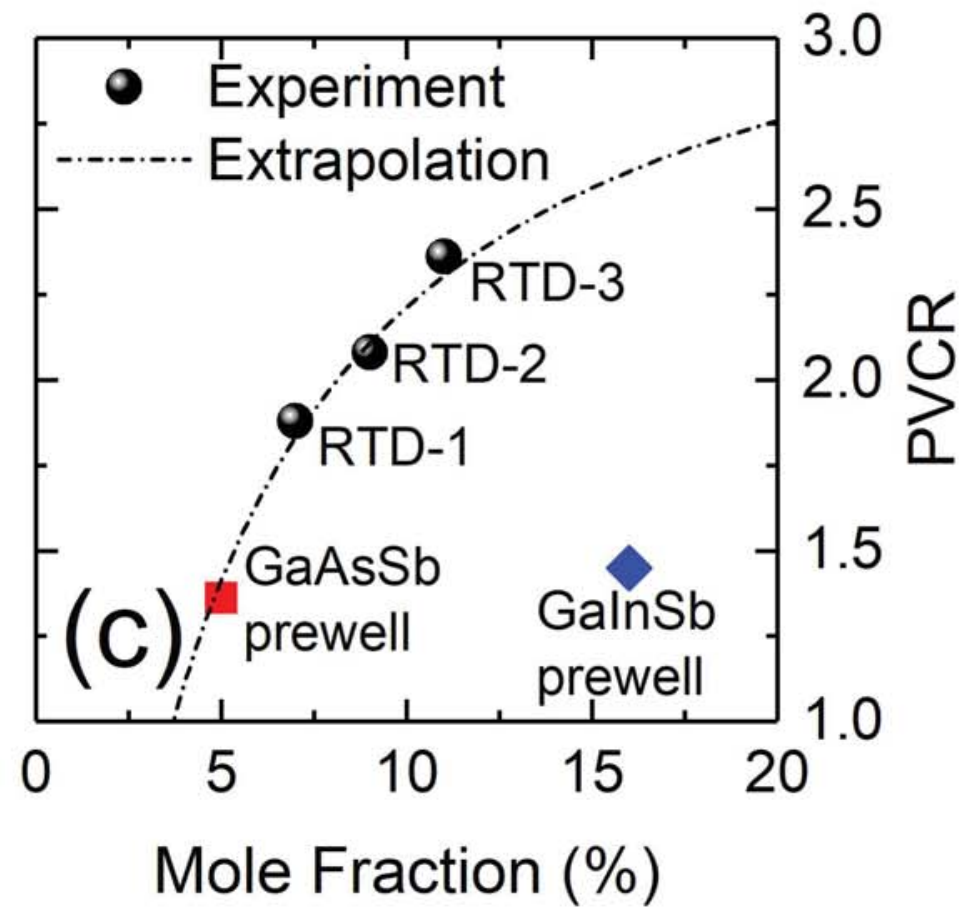



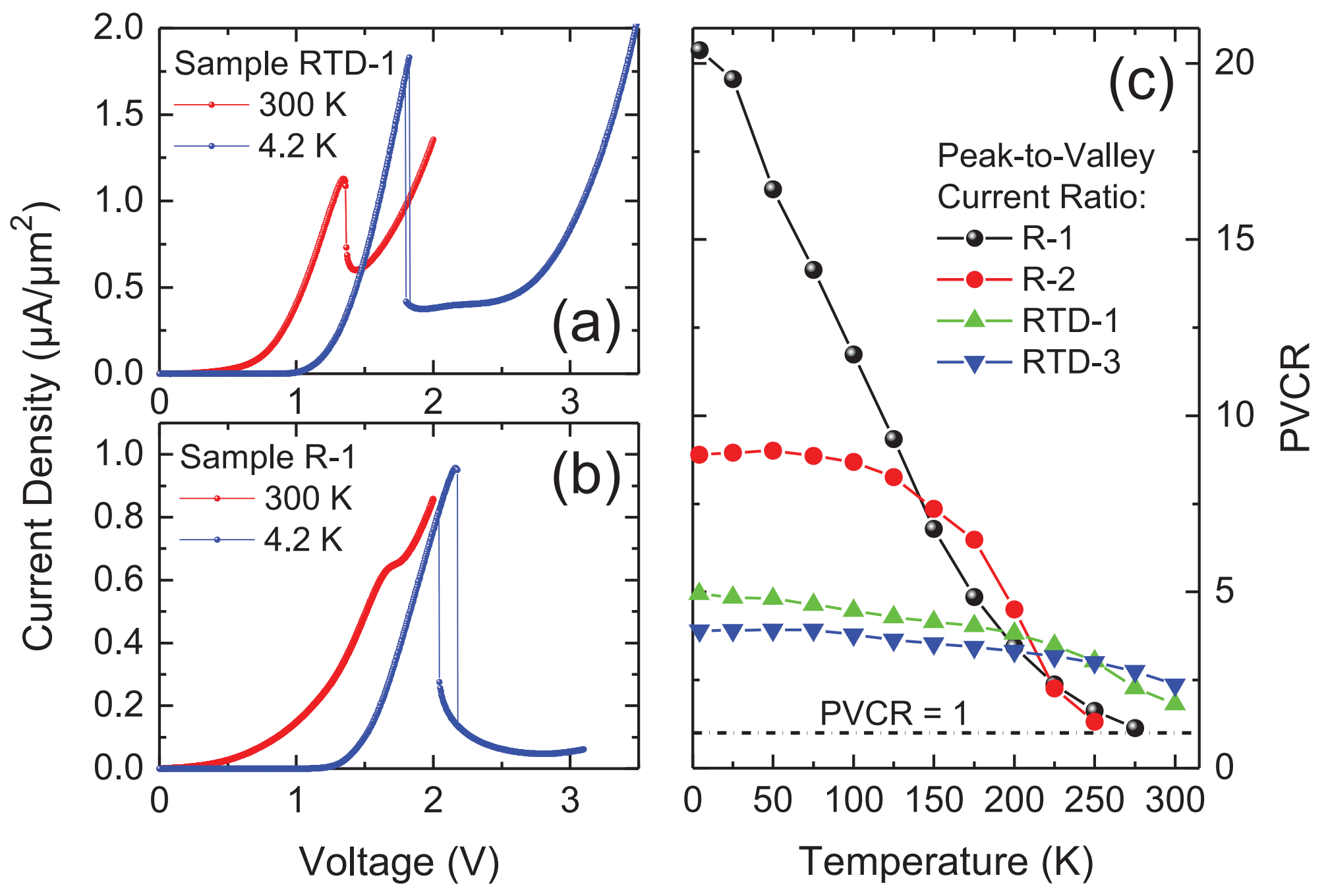